\title{
A double-blind, randomised trial of a polyphenolic-rich nail bed balm for chemotherapy-induced onycholysis: the UK polybalm study
}

\author{
Robert Thomas $^{1,2,5} \cdot$ Madeleine Williams $^{2} \cdot$ Michael Cauchi $^{3} \cdot$ Saul Berkovitz $^{4} \cdot$ Sarah A. Smith $^{1}$
}

Received: 4 April 2018 / Accepted: 10 April 2018 / Published online: 7 May 2018

(c) The Author(s) 2018

\begin{abstract}
Purpose Nail damage is common amongst patients receiving chemotherapy causing disfigurement and pain. This investigation evaluated whether a topical balm containing steam-extracted, bioactive polyphenolic-rich herbal oils blended with organic waxes could protect the nails via their reported anti-inflammatory, analgesic, anti-oxidant and anti-microbial properties.

Methods 60 patients (23M, 37F) were randomised to apply (2-3/day) either the plant balm (PB) or a petroleum control (PC) to their nail beds. Demographics, type and number of chemotherapy cycles did not differ between the two groups, recruited between Sept 2015 and Sept 2016. An unpaired $t$ test was used to test the differences in symptoms and physical nail damage between the two groups.

Results Symptom scores recorded with the dermatology life quality questionnaire (DLQQ) were significantly better, between the start and end of chemotherapy, in the group applying the PB versus PC. Likewise, the mean fall in nail damage, scored with the Nail Psoriasis Index by the supervising physician, was also significantly different.

Conclusion The polyphenolic-rich essential oils and plant-based waxes in this nail bed balm profoundly reduced chemotherapy-related nail damage and improved nail-related quality of life, compared to a control. A further analysis is planned combining this balm with nail bed cooling.
\end{abstract}

Keywords Prevention of onycholysis · Breast cancer · Prostate cancer · Taxane chemotherapy side effect · Quality of life · Randomised · Double-blind clinical trial

\section{Discussion and implications for clinical practice}

Scientific evidence for practical strategies to reduce this distressing and unsightly side effect of one of the most commonly used chemotherapy drugs is long overdue. Up until

Robert Thomas

rt@cancernet.co.uk; robert.thomas@bedfordhospital.nhs.uk

1 Bedford and Addenbrooke's Cambridge University Hospital Trusts c/o The Primrose Unit, Kempston Rd, Bedford MK42 9DJ, UK

2 The Primrose Unit Research Office, Bedford Hospital, Kempston Road, Bedford MK42 9DJ, UK

3 Department of Mathematics and Statistics, University of Limerick, Limerick, Ireland

4 Royal London Hospital for Integrated Medicine, Great Ormond Street, London WC1N 3HR, UK

5 Department of Biological and Exercise Science, Coventry Univerity, Priory Street, Coventry CV1 5FB, UK now, many patient advocacy groups have advised patients to use dark nail varnish or standard petroleum-based nail balms to an attempt to help protect their nails during chemotherapy, but there is little published evidence for their effectiveness or even anecdotal reports of helping [1]. Cooling the nails during chemotherapy has been shown to reduce nail toxicity but commercially available gloves are rarely used in the UK, as nurses have practical concerns about restricted access to patients' hands. The profoundly lower levels of physical nail damage and improvements in nail-related quality of life in the cohort applying the plant-based balm in this robustly designed RCT strongly suggest that this balm will benefit future patients receiving chemotherapy in route clinical practice. As nail toxicity still occurred in a minority of participants, who had marked general side effects of chemotherapy, further research combining this balm with cooling would be justified. 


\section{Introduction}

Chemotherapy-induced onycholysis is caused by disorganised inhibition of the rapidly dividing cells in the nail bed [2-5]. Further contributory factors relate to the anti-angiogenetic properties of taxanes and excess local inflammatory mediated processes [2-5]. Disruption of the normal anatomy allows bacterial and fungal pathogens to enter the nail beds causing further damage $[2,5]$. In most cases this manifests as ridges in the nails that correspond to the timings of chemotherapy episodes, known as Beau's lines (Image 1). More pronounced nail damage, particularly associated with taxanes, can lead to discolouration, painful subungual haemorrhages (Image 2), distortion and complete onycholysis which is distressing, unsightly, affects body image (Images 3, 4), limits activities of daily living and may lead to more serious consequences including secondary infection which is a particular concern amongst patients at risk of neutropenia [2-6].

Cooling the fingers has been reported to reduce docetaxel-related nail toxicity by initiating vasoconstriction and slowing the metabolism of onychocytes [7-9]. This technique, however, has not been commonly adopted in the UK, as oncology nurses have concerns about restricting access to patients' hands. Patient support groups advise a variety of anecdotal strategies including wearing nail varnish and massaging moisturising petroleum-based balms into the nail bed, but there have been no robust prospective studies to support these practices [1].

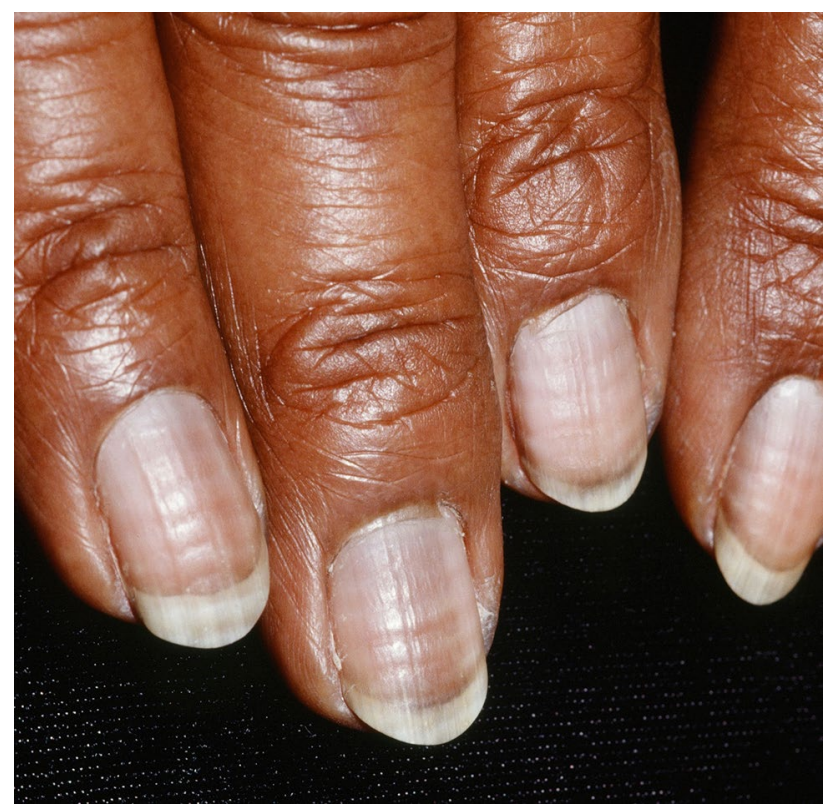

Image 1 Mild, asymptomatic nail changes corresponding to the cycles of chemotherapy-Beau's Lines

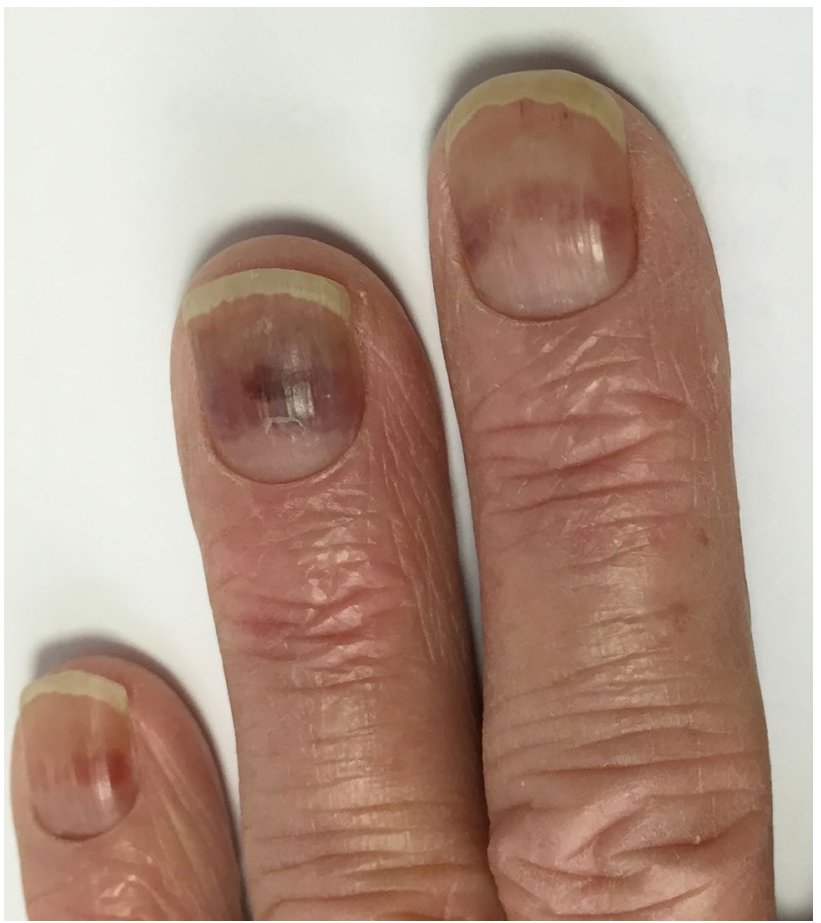

Image 2 Uncomfortable, chemotherapy induced subungual haemorrhages

The plant-based waxes and essential oils selected for this study are naturally rich in phytochemicals, especially the phenolic polyphenols group which are reported to have anti-oxidant, anti-inflammatory, DNA repair enhancing and anti-microbial properties [10-17]. In addition to their abilities to moisturise the skin, preventing drying and the splitting of nails, it was hypothesised, they would be sufficiently absorbed into the nail beds to act as a local antidote to the chemotherapy, preventing damage to the proliferating stem

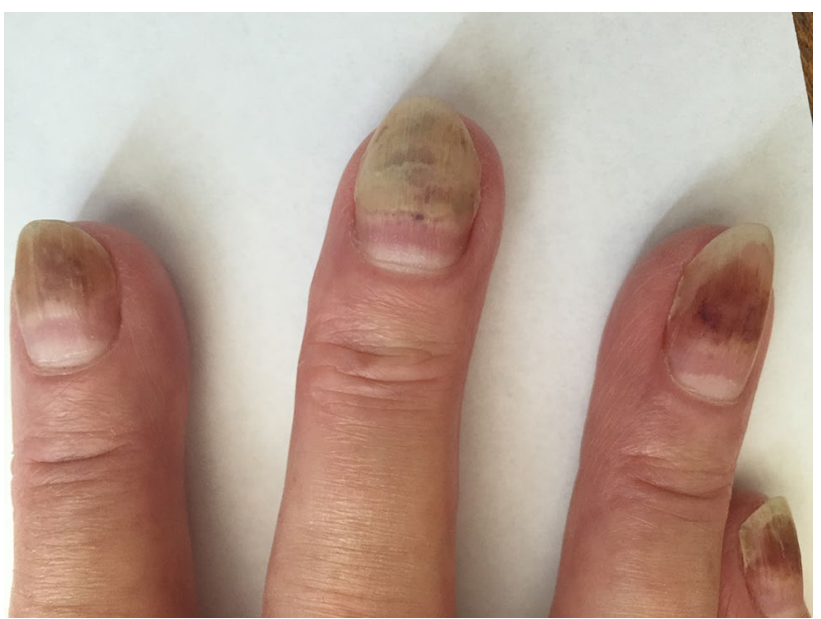

Image 3 Painful chemotherapy induced parial onycholyis 


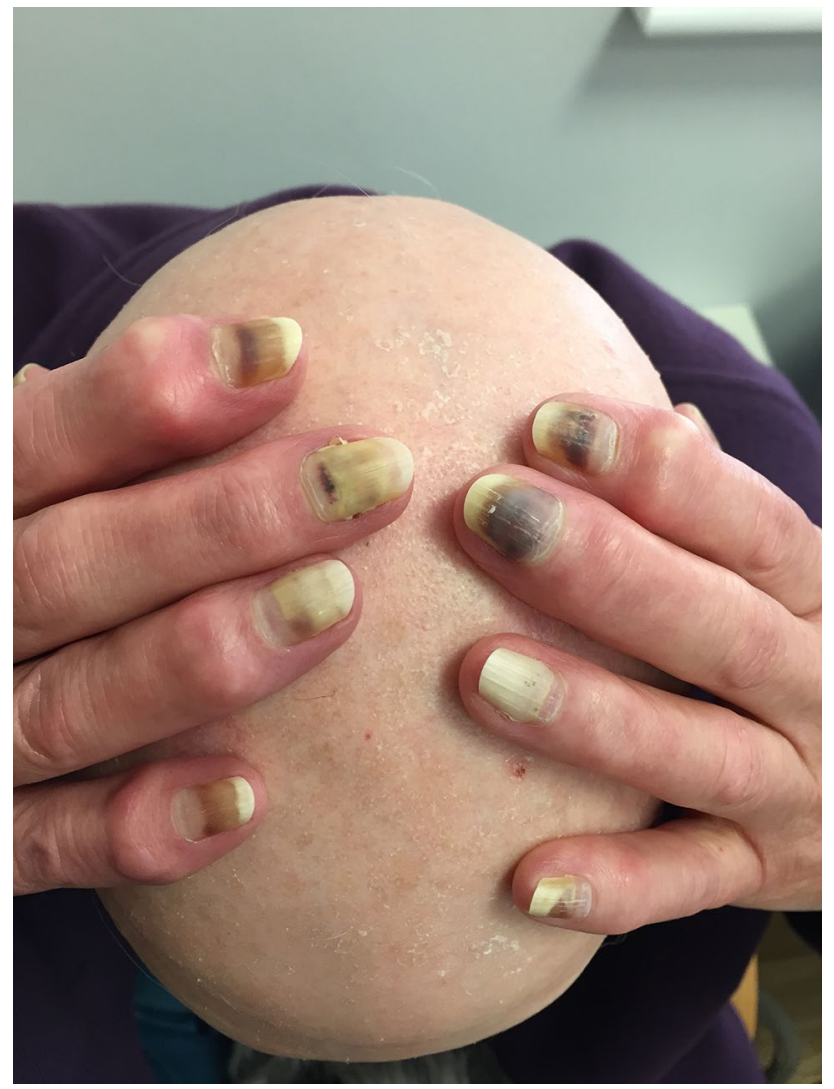

Image 4 Severe, distressing subungual haemorrhages and secondary infection leading to complete onycholysis

cells as well as avoiding secondary damage from inflammation or secondary infection [10-19].

\section{Methodology}

The study recruited for 13 months at one of Cambridge University's affiliated hospitals, Bedford Hospital. In total, 23 men with prostate cancer and 37 women with breast cancer receiving chemotherapy were randomised from a total of 63 patients considered for this study, between September 2015 and October 2016. One patient declined entry after reading the information materials and two had pre-existing nail conditions [See consort diagram]. Following written informed consent, randomisation occurred when the supervising physician contacted the trials office who then opened the next sealed envelope in strict numerical order. The randomised groups were demographically equally matched in terms of number of chemotherapy cycles received, gender, chemotherapy type, baseline nail health and age (see Table 1). The balms were supplied in $50 \mathrm{ml}$ pots, labelled A or B, together with an applicator. They were similar in colour, consistency
Table 1 Demographics

\begin{tabular}{llll}
\hline & Plant balm & Placebo & Statistical difference \\
\hline Number for analysis & 30 & 30 & $0(\mathrm{~ns})$ \\
Average age & 57.2 & 66.3 & $9.1(\mathrm{NS})$ \\
Sex (women:men) & $18: 11$ & $19: 12$ & $1(\mathrm{~ns})$ \\
Average chemo cycles & 4.4 & 5.2 & $0.8(\mathrm{~ns})$ \\
Received taxotere & 28 & 29 & $1(\mathrm{~ns})$ \\
\hline
\end{tabular}

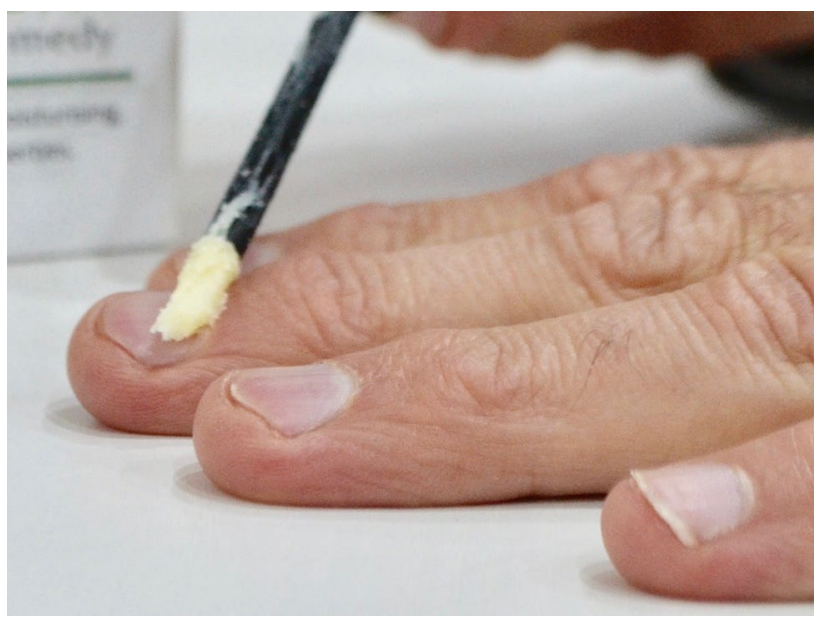

Image 5 Polybalm applied to cuticles, surrounding skin and nail beds on the fingers preserving nail health

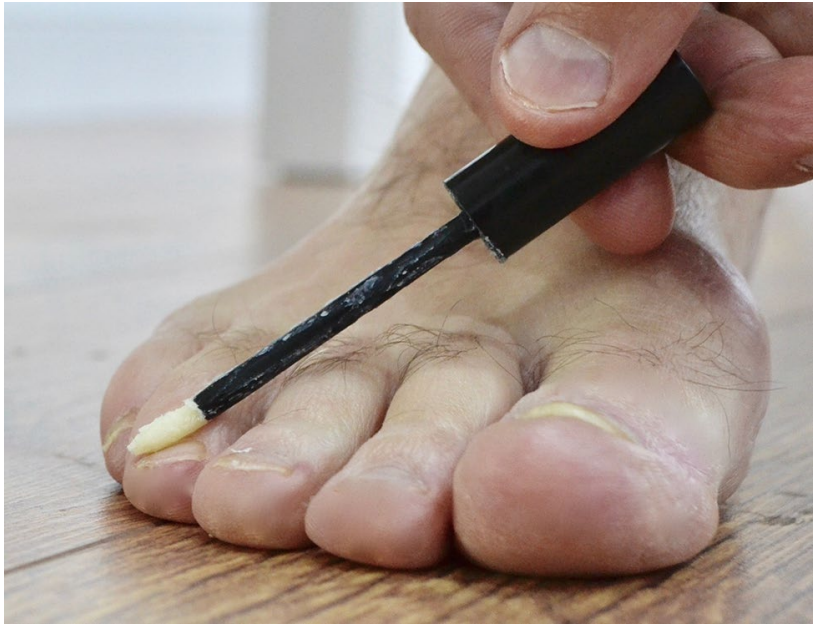

Image 6 Polybalm applied to cuticles of toe nails

and smell. Participants were shown how to apply their allocated balm to their finger nail beds, $2-3$ times a day, until the end of their chemotherapy (Images 5, 6). No members of the research team, physicians, participants or the statistician were aware of the contents of either balm pot until the final results were analysed. 


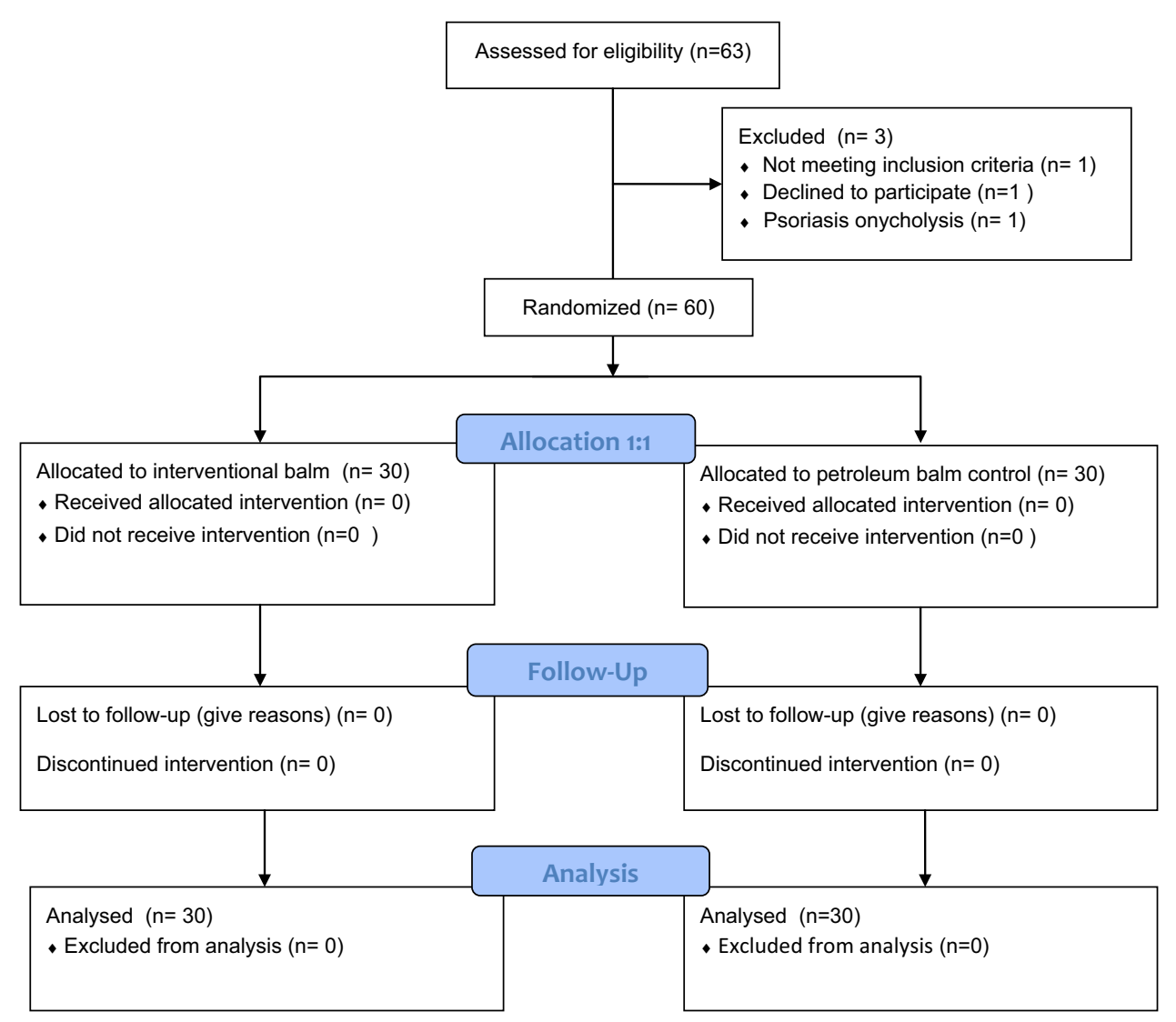

At baseline and at the end of chemotherapy, patients recorded symptoms relating to their nail health using the validated Dermatology Life Quality Questionnaire plus a Linear Severity Scale (LSS). Physicians recorded the physical appearance of the nails using the validated Nail Psoriasis Severity Index (NPSI) plus the Linear Severity Scale. Photographs of the nails were also sent to further three independent physicians for external validation.

\section{Ingredients of the balms}

The PB consisted of a blend of unrefined shea butter (Butyrospermum parkii), organic beeswax (Cera alba), extra virgin, organic, cold-pressed olive oil (Olea europaea), organic cocoa seed butter (Theobroma cacao), African sage
(Tarchonanthus camphoratus), wintergreen leaf (Gaultheria procumbens), lavender flower (Lavandula spica) and eucalyptus leaf (Eucalyptus globulus). The oils were steam extracted from the plants and then gently blended, avoiding overheating to prevent damage to the phytochemicals. They contained no preservatives, parabens, sulphates or perfumes. The placebo control balm contained hydrogenated petroleum oil, candela wax, lavender and eucalyptus essences to ensure their placebo effect.

\section{Quality assurance}

The trial was designed with the help of the independent National Cancer Research Clinical Trials Development Committee as well as oncologists from Addenbrooke's Hospital, advisors from the Department of Biological Science
Table 2 Summary of average chance in score at baseline and end of study

\begin{tabular}{lllll}
\hline Nail health record & Plant Balm & Placebo & Difference (CI) & Power \\
\hline Dermatology life quality questionnaire & -0.034 & -6.10 & 6.06 CI 4.17-7.95 & $p<0.00001$ \\
Linear severity scale (mm) & +2.63 & -64.1 & 66.72 CI 52.97-80.47 & $p<0.00001$ \\
Nail Psoriasis Index (NPSI) & 0.0 & -5.71 & 5.71 CI 4.29-7.12 & $p<0.00001$ \\
Linear analogue scale (mm) & -5.79 & -66.1 & 60.30 CI 45.29-75.32 & $p<0.00001$ \\
\hline
\end{tabular}


at Coventry University and an independent statistician. The study was externally audited to comply with Good Clinical Practice (GCP) guidelines, and East of EnglandCambridge Central Research Ethics Committee standards (REC reference: 15/EE/0357). The investigational balms were made specifically for this study by Power Health Ltd, Yorkshire. Additional stability and microbial tests of the formula were performed by Advantis Laboratories Ltd, to comply with European Union Cosmetics Standards (REC Ref: 76/768/EEC).

\section{Statistical considerations}

The sample size was determined considering a previously reported incidence of chemotherapy-induced onycholysis of between 40 and $70 \%$ [2-5]. The trial development committee deemed that deterioration in nail toxicity score of $20 \%$ was clinically significant. For the power calculation, the nearest relevant trial using similar toxicity grading scores evaluated the benefits of a frozen glove on the incidence of onycholysis which had 20 and 25 patients, respectively, to produce an $80 \%$ statistical power for a two-sided test at the $\alpha=0.05$ level. A Matlab 2011a calculation was employed to determine the sample size. The resultant graph estimated that 50 patients would have been adequate to detect a $20 \%$ difference in toxicity with a $\sqrt{ } 2 *$ standard deviation (SD) of confidence of using a two-sided test at the $\alpha=0.05$ level. The trial committee therefore decided on 60 participants to allow unforeseen events or dropouts. The analysis compared the difference in nail damage scores between the start of the study and end of chemotherapy, and between the plant balm and control groups, using an unpaired $t$ test for all four measures of nail health with a significance level of $\alpha=0.05$ at $95 \%$.

\section{Results}

Patient and physician measures of nail toxicities demonstrated large and statistically significant differences between the plant balm (PB) and placebo control (PC) groups (Table 2). In the PB group, the DLQQ score dropped from baseline to the end of the chemotherapy by an average of 0.034 . In the PC group, the DLQQ score dropped by an average of 6.10. This resulted in a mean difference in the level of deterioration of quality of life of 6.06 , confidence intervals (CI) 4.17-7.95, a significance of $p<0.00001$ using an unpaired $t$ test. For the linear severity scale (LSS) the average condition of the nails recorded by the patients actually improved over the course of chemotherapy by $2.63 \mathrm{~mm}$ in the PB cohort compared to a deterioration of $64.1 \mathrm{~mm}$ in the PC group (difference $66.72 \mathrm{~mm}$; CI 52.97-80.47, $p<0.00001)$. Difference in the physical appearances of the nails was similarly large and statistically significant measured using the validated NAPSI as well as a Standard Linear Analogue Scale. The figures for the other measures of nail health are summarised in Table 2.

It was interesting to note that the two patients in the plant balm group who still had moderate damage, also suffered from other severe chemotherapy complications including neutropenic sepsis, diarrhoea and peripheral neuropathy. There were no reported balm-related adverse toxicities although one patient in the plant balm group discontinued use before the end of the first cycle, quoting time constraints as the reason for stopping.

\section{Discussion}

The phytochemical-rich essential oils and plant-based waxes in this nail bed balm considerably reduced the physical appearance and distress of chemotherapy-induced nail damage compared to a petroleum-based control balm. In terms of trial design, it may have seemed more relevant to have considered a within patient comparison, applying one cream to one hand and the other to the opposite hand. However, this was dismissed as impractical by the design committee, as breaches in compliance would have overridden any statistical benefits as participants would very likely have mixed up the allocated hands and as soon as any difference were realised. The second design issue was the choice of placebo control. As there were no published nail bed balm data available at the start of the trial, a statistically valid comparison would be an investigational balm versus no balm. This, however, would have meant that the trial would not have been blind; so a simple, commonly used, petroleum-based balm was chosen, with added essences determined by the manufacturers. It was assumed that the control may have provided some benefit via its moisturising properties, but not have the potential additional properties of the plant oils and waxes.

Previous studies have reported that regular oral intake of polyphenol-rich foods and supplements has demonstrated skin protection from sun damage, and lower the risk of chronic inflammatory diseases including arthritis, diabetes and cancer as well as improved recovery after exercise [20-23]. The potential benefits of topical phytochemicalrich plant extracts have been explored in laboratory studies, but only in a limited number of clinical trials despite their 
popular inclusion in skincare cosmetics $[15,24]$. They have not previously been investigated in the onycholysis setting. This study did not elucidate which of the reported bioactive pathways of these phytochemicals provided the most benefit, but there are several potential possibilities.

Their anti-oxidative properties could have potentially protected the rapidly proliferating onychocytes from oxidative damage [12, 13, 15-17]. Gaultheria procumbens, particularly high in methyl-salicylate, may have reduced an inflammation reaction against the nail bed tissues initiated by local chemotherapy damage, preventing further structural damage. These properties may have reduced discomfort via their local analgesic properties $[11,14,25]$. Tarchonanthus camphoratus, containing phenolic acids, flavonoids and tannins, has also been shown to have anti-inflammatory properties with potency twice as high as that of indomethacin. In addition, its bactericidal and fungicidal properties could have protected the nail bed from secondary infection, especially if splits or cracks had occurred [25, 26]. Both Lavandula officinalis and Eucalyptus globulus contain lactones, flavonoids, tannins, phenolic acids and terpene esters, which also have anti-inflammatory, anti-fungal and bacteriocidal properties $[25,26]$. The base waxes create a protective barrier for skin which helps it to retain moisture, preventing splitting and cracking of the nail bed which could allow entry of pathogens $[24,26]$. Unlike the standard petroleum-based waxes, they also have bioactive properties of their own.

Olea europaea, predominantly oleic acid, has anti-oxidant and anti-inflammatory properties [24] and has demonstrated, in a randomised laboratory study, to reduce epidermal levels of 8-hydroxy-deoxyguanosine, a marker for DNA damage after animals were exposed to excess sunlight [15]. Theobroma cacao and Butyrospermum parkii, approved by the FDA for use as natural bases in cosmetics, have anti-oxidant properties via their vitamin $\mathrm{E}$ and $\mathrm{A}$ content, and even after processing, cera alba has anti-bacterial and anti-fungal properties $[24,26]$. Although there are some overlaps in these properties, by including different plant extracts, each with a range of phytochemicals, it was hypothesised that their different reported modes of action would have a synergistic protective affect [24-26].

In conclusion, the large difference between the control and interventional balms in this double-blind randomised study strongly suggests that the phytochemicals in natural oils and waxes were sufficiently absorbed into the nail beds to enable their bioactive properties to protect participants from the harmful effects of chemotherapy. In this study, participants applied the balm at the start, or near the start of chemotherapy, so the study did not answer the research question of whether this balm could prevent further deterioration, or provide relief once damage had occurred. Precisely which properties and of which ingredient had the most relevant benefit, was not answered in this study, and further research in this field is certainly justified, as this could lead to enhanced versions of this and other topical therapies. Further research, combining different versions of balm with or without nail cooling, is planned to evaluate whether this will further protect patients from the distress of onycholysis.

Acknowledgements Thanks to the Provincial Grand Lodge of Bedfordshire, who provided financial support for this study. No member of the research team received payments to recruit patients into the study. Thanks to the scientific committee and detailed advice from the members of the National Cancer Research Clinical Trials Development Committee. Thanks to Power Health Ltd, Yorkshire, who made the balms specifically for this study. Although this was a scientific evaluation, Medicines and Health Regulatory Authority (MHRA) classed these products as a cosmetic, so cannot be recommended for any medical condition or claim health benefits. The investigation balm should not be used against medical advice. The protocol was in the public domain and the balm, named after the clinical trial, is now distributed by an independent organisation (polybalm.com) that has no connection to the trials unit.

Open Access This article is distributed under the terms of the Creative Commons Attribution 4.0 International License (http://creativeco mmons.org/licenses/by/4.0/), which permits unrestricted use, distribution, and reproduction in any medium, provided you give appropriate credit to the original author(s) and the source, provide a link to the Creative Commons license, and indicate if changes were made. 


\section{Appendix: A double blind, randomised evaluation of nail bed balms to prevent chemotherapy-induced onycholysis-The polybalm study}

Patient Initials Trial No.:

Date:

Please indicate treatment cycle:

\begin{tabular}{|l|l|l|l|l|l|l|l|l|l|}
\hline Baseline & C2 & C3 & C4 & C5 & C6 & C7 & C8 & C9 & C10 \\
\hline & & & & & & & & & \\
& & & & & & & & & \\
\hline
\end{tabular}

Over the last seven days, please circle the response which you feel best answers the questions about your fingernails.

Not at all $\begin{gathered}\text { A little } \\ \text { bit }\end{gathered} \quad$ Somewhat $\begin{gathered}\text { Quite a } \\ \text { bit }\end{gathered} \quad \begin{gathered}\text { Very } \\ \text { much }\end{gathered}$

1 I have pain in my fingernails.

2 I have redness and swelling around my fingernails and the nail bed.

3

The condition of my nails limits my daily activities.

$4 \quad$ I am upset by the appearance of my nails.
0

0

0

0
1

1

1

1

2
4

4

4

4

Please hand this questionnaire back to a member of the research team. Thank you.

\section{References}

1. Macmillan (2018) Caring for skin and nails. https://www.macmi llan.org.uk/information-and-support/coping/changes-to-appea rance-and-body-image/changes-during-treatment/caring-for-skinand-nails.html. Accessed Mar 2018

2. Piraccini BM, Alessandrini A (2013) Drug-related nail disease. Clin Dermatol 31:618-626

3. Minisini AM, Tosti A, Sobrero AF et al (2003) Taxane-induced nail changes: incidence, clinical presentation and outcome. Ann Oncol 14:333-337

4. Battegay EJ (1995) Angiogenesis: mechanistic insights, neovascular diseases, and therapeutic prospects. J Mol Med 73:333-346

5. Wasner G, Hilpert F, Schattschneider J et al (2002) Docetaxelinduced nail changes: a neurogenic mechanism-a case report. J Neurooncol 58:167-174
6. Eisman S, Sinclair R (2014) Fungal nail infection: diagnosis and management. BMJ 348(7953):27-32. https://doi.org/10.1136/bmj. g1800

7. Ding PN, Thomas RJ (2010) A cool solution for docetaxel induced onycholysis. Focus Cancer Med 2(1):18-19

8. Scotté F, Tourani J-M, Banu E et al (2005) Multicenter study of a frozen glove to prevent docetaxel-induced onycholysis and cutaneous toxicity of the hand. J Clin Oncol 23(19):4424-4429

9. Ishiguro H, Takashima D, Yoshimura K et al (2012) Degree of freezing does not affect efficacy of frozen gloves for prevention of docetaxel-induced nail toxicity in breast cancer patients. Support Care Cancer 20:2017-2024

10. Delaquis PJ, Stanich K, Girard B (2002) Antimicrobial activity of individual of essential oils. Int J Food Microbiol 74(1-2):101-109

11. Dawid-Pać R (2013) Medicinal plants used in treatment of inflammatory skin diseases. Postepy Dermatol Alerqol 30(3):170-177 
12. Smith-Palmer A, Stewart B, Fyfe C (2002) Antimicrobial properties of plant essential oils and essences against five important food-borne pathogens. Lett Appl Microbiol 26(2):118-122

13. Korać RR, Khambholja KM (2011) Herbs protect cells from UV radiation. Pharmacogn Rev 5(10):164-173

14. Man M-Q, Hupe M, Sun R et al (2012) Topical apigenin alleviates cutaneous inflammation in murine models. Evid Based Complement Altern Med 2012:912028. https://doi. org/10.1155/2012/912028

15. Ichihashi M, Ahmed NU, Budiyanto A et al (2000) Preventive effect of antioxidant on ultraviolet-induced skin cancer in mice. $\mathbf{J}$ Dermatol Sci 23:S45-S50

16. Tiziana Baratta MT, Damien Dorman HJ, Deans SG et al (2001) Antimicrobial and antioxidant properties of some commercial essential oils. Flavour Fragr J 13(4):235-244

17. Baliga MS, Katiyar SK (2006) Chemoprevention of photocarcinogenesis by selected dietary botanicals. Photochem Photobiol Sci 5(2):243-253

18. Milin C, Domitrović R, Tota J et al (2001) Effect of olive oil and corn oil enriched diets on the tissue mineral content in mice. Biol Trace Elem Res 82(1-3):201-210

19. Jensen CD, Andersen KE (1999) Allergic contact dermatitis from cera alba (purified propolis) in a balms. Contact Dermatitis $55: 312-313$
20. Afaq F, Katiyar SK (2011) Polyphenols: skin photoprotection and inhibition of photocarcinogenesis. Mini Rev Med Chem 11(14):1200-1125

21. Kim Y, Keogh JB, Clifton PM (2016) Polyphenols and glycemic control. Nutrients. https://doi.org/10.3390/nu8010017

22. Thomas R, Williams M, Sharma $\mathrm{H}$ et al (2014) A double-blind placebo-controlled randomised trial evaluating the effect of a polyphenol-rich whole food supplement on PSA progression in men with prostate cancer the UK National Cancer Research Network (NCRN) Pomi-T study. Prostate Cancer Prostatic Dis $17: 180-186$

23. Thomas R, Stacey K, Jimenez A (2017) Exercise-induced biochemical changes and their potential influence on cancer: a scientific review. Br J Sports Med 51:640-644

24. Stallings AF, Lupo M (2009) Practical uses of botanicals in skin care. J Clin Aesthet Dermatol 2(1):36-40

25. Blumenthal M, Goldberg A, Brinckmann J (2000) Herbal medicine: expanded commission e monographs. Integrative Medicine Communications, Boston, pp 330-333

26. Food and Drug Administration HaHS (2003) Skin protectant drug products for over-the-counter human use; final monograph: final rule. Fed Reg 68:33362-33381 